\title{
Bibliografía sobre John Dewey: Filosofía de la educación
}

\author{
PACIANO FERMOSO ESTÉBANEZ \\ Universidad Autónoma de Barcelona
}

\begin{abstract}
SUMMARY.-In this article the autor selects the J. Dewey's original writings and the most important bibliography about his educational thought. Before his writings and before the general bibliography are a brief biography, and introduction to his philosophical thought, his main philosophical works and some monographic studies about his philosophical conception.

His tehoric/philosophical educational thought and his educational writings are the first essence of this deweyian bibliography.

In the last section ar classified in seven paragraphs all books and articles about the educational thought of J. Dewey: general studies and repertoires; foundatios of his educational theory; philosophy and education; his influence in the educational world; monographic studides; relations with the philosophical systems; and others.
\end{abstract}

La Bibliografía presente no abarca toda la producción del propio John Dewey, ni todo cuanto sobre él se ha escrito, considerada su polifacética personalidad. Me circunscribo únicamente a sus escritos pedagógicos y a su repercusión en la investigación filosófica sobre educación. Más aún, omito las obras, que no tengan nivel epistemológico filosófico; es decir, me limito a su Filosofía de la Educación.

\section{BIOGRAFÍA}

John Dewey nació (1859) en Burlington, Estado de Vermont, en cuya Universidad estatal conoció el pensamiento evolucionista y el positivismo comtiano. En 1884 se doctoró en Filosofía, en la Universidad de John Hopkins de Baltimore, donde fue iniciado en el neoidealismo hegeliano, versando su tesis sobre la psicología de Kant. Ejerció su profesorado en varias universidades norteamericanas. En primer lugar, enseñó en la Universidad de Michigan (1884-1894); tras una breve estancia en la Universidad de Minnesota, pasó a la de Chicago (1894-1904), en la que organizó, junto con su esposa Alice Chipman, una escuela experimental, para someter a prueba sus teorías pedagógicas. Finalmente, la Columbia University de New York se benefició de su docencia desde 1905 hasta 1929, fecha de su jubilación; fueron 
los años más intensos bibliográficamente. Viajó, dando conferencias, por diversos países europeos, por Japón, China y Rusia. Como psicólogo fue uno de los principales representantes del Funcionalismo norteamericano; como filósofo fue pragmatista; y como teórico de la educación creó el movimiento del «Progresismo». Falleció en 1952.

\section{JOHN DEWEY COMO FILÓSOFO}

\subsection{Su pensamiento filosófico}

Se han sugerido ya sus raíces: pragmatismo de W. James; neoidealismo hegeliano; y evolucionismo darwiniano. Pronto abandonó las tesis idealistas, conservando siempre la impronta de las otras dos influencias.

Su filosofía resultaría incomprensible, si se la desconectase de su teoría educacional, pues «la filosofía podía casi definirse como el pensar que ha llegado a ser consecuencia de sí mismo, que ha generalizado su lugar, función y valor de la experiencia» ${ }^{1}$; «la filosofía puede, incluso, definirse como la teoría general de la educación $»^{2}$. A pesar de todo, ha de buscarse el fundamento del pensamiento deweyano educacional en la Metafísica, en la Antropología, en la Epistemología, en la Axiología y en la Etica.

Las influencias recibidas se reflejan abiertamente en su Epistemología, cuya idea central es la experiencia, la cual es fuente de riesgo y de vaivenes en la existencia humana. La Epistemología de Dewey, como es lógico, está, a su vez, condicionada por la Metafísica. Las realidades sustanciales no existen; y más allá de la experiencia el hombre transforma la naturaleza y accede al conocimiento, a través del cual el hombre modifica a aquella, despojándola de la inestabilidad que le es inherente. Los fenómenos naturales están concatenados entre sí, formando verdaderas transacciones regidas por la ley de la adaptación. La naturaleza es el conjunto estructurado de transacciones; en ella todo es cambiante, como sometida a la evolución constante.

Dewey pretendió poner dique al intelectualismo e incrementar la ambición inventora de las ciencias experimentales. El pensamiento sólo tiene sentido, si se une a la acción, pues se origina en la formulación de hipótesis para resolver las dificultades. El conocimiento es el instrumento para perfeccionar las realidades y será tanto más acertado cuanto mayores sean los resultados - the practical outcome-. El conocimiento busca destruir la irracionalidad y de esta manera surgen la ciencia y la filosofía.

La investigación es el núcleo primero del pensamiento lógico; por ella se restablece el equilibrio en la naturaleza inestable y cambiante. Cuando se soluciona un problema, hemos concebido una idea, y ésta es una previsión de la nueva experiencia.

En Antropología John Dewey superó el dualismo cartesiano, ya que el pensamiento y la materia son cualidades de la misma realidad. El hombre no es ni alma

1. J. Dewey (1971), Democracia y educación, Losada, Buenos Aires, p. 345.

2. J. DeWEY, o. c., p. 347. 
ni yo personal, sino una agrupación de fenómenos y de actividades. El hombre es cambio continuo, al que únicamente pone freno la adaptación al medio. La conciencia es el cambio mismo, es una sensación constante de duda, que pide una nueva adaptación. El hombre es una realidad corpórea, vital, mental y social; las discusiones sobre las relaciones entre el alma y el cuerpo son cuestiones bizantinas. El funcionalismo de J. Dewey es una concepción psicológica holística, contrapuesta al estructuralismo atomista de Titchener.

La Axiología no puede ser universalista y absolutista, porque se lo impide su Metafísica. La axiología es relativista y cambiante. Los valores puede ser intrínsecos —inabordables por el juicio- y extrínsecos, que permiten comparar, estimar y apreciar, por lo que bien pueden ser llamados valores instrumentales; y son los únicos jerarquizables. Los valores no pueden ser fijos, dado el «rápido cambio de los intereses políticos, científicos y económicos del último siglo»³.

Intimamente unida a su axiología está la Etica, cuya estructura gira en torno a los valores morales, que son reflejo de un ideal noble, estabilizador de las normas de acción y unificador del egoísmo y de las pasiones. No puede haber valores éticos absolutos en una metafísica intrascendente. Los valores, como la realidad, cambian; y la moralidad consiste en el hallazgo de nuevas formas de obrar, con mayor equilibrio social. La Etica es inseparable de sus convicciones democráticas y de su interpretación social de la vida.

\subsection{Principales obras filosóficas de John Dewey}

DEWEY, J. (1903): Studies in logical Theory, Chicago.

- (1908): Ethics, G. Bell and Sons, New York.

- (1910): How we think, Boston. Traduc. Castellana: «Cómo pensamos», La Lectura, Madrid, 1920. Verdadero tratado de Epistemología. Sin su lectura es difícil entender el pensamiento pedagógico de Dewey.

- (1920): Reconstruction and Philosophy, Traduc. Castellana: «Reconstrucción de la conducta», Losada, Buenos Aires, 1955.

- (1922): Human Nature and Conduct, Holt, New York. Traduc. Castellana: «Naturaleza humana y conducta», F.C.E., México, 1964.

- (1929): The Quest for Certainty, Balch and Co., Minton. Traduc. Castellana: «La búsqueda de la certeza», F.C.E., México, 1952.

- (1935): Logic: The theory of inquiry, New York. Traduc. Castellana: «La lógica de la investigación», F.C.E., México, 1946.

- (1939): Theory of Valuation, University of Chicago Press.

- (1946): Problem of Men, New York. Traduc. Castellana: «El hombre y sus problemas», Losada, Buenos Aires, 1952.

- (1969 y ss.): The collected Works of J. Dewey: 1882-1953, Sothern Illinois University Press, Carbondale. Editadas por A. Boydston. La edición completa constará de 31 volúmenes.

- The Earley Works: 1882-1898, 5 Volúmenes ya publicados.

- The Middle Works: 1899-1924, 10 Volúmenes ya publicados.

- The Later Works: 1925-1953, 8 Volúmenes publicados hasta 1986. El total de este último período abarcará 16 Volúmenes.

3. J. DewEY, o. c., p. 263. 


\subsection{Introducción a sus obras filosóficas}

BELlARATE, B. (1979): "Tornare a John Dewey? Spunti in margine a una nuova edizione dei soi scritti», Orientamenti Pedagogici, Vol. 26, nro. 4, pp. 732-736.

BoYDSTON, J. A. (Ed.) (1970): Guide to the works of John Dewey, Southern Illinois University Press, Carbonale.

Boydston, J. A.; ANDERSEN, R. L. (1969): J. Dewey: A checklist of translations 19001967, Southern Illinois University Press. Interesante Repertorio sobre las traducciones a distintos idiomas de las obras originales de J. Dewey. Puede ayudar a comprender el alcance del círculo de influencia.

BOYDSTON, J. A.; POULES, K. (1974): Checklist of writings about J. Dewey. De interés para los especialistas en esta dimensión de su producción, por ser un Repertorio de la bibliografía acerca de su pensamiento filosófico.

\subsection{Algunas monografías sobre el pensamiento filosófico de J. Dewey}

Berger, G. (1952): La Philosophie de J. Dewey, París.

BURTT, E. A. y otros (1960): J. Dewey: His thought and influence.

CAHN, S. M. (Ed.) (1977): New studies in the Philosophy of J. Dewey, University Press of New England, Hanover (N. H.).

DAL PRA, M. (Ed.) (1952): Il pensiero di J. Dewey, Bocca, Milano.

Dykhuizen, G. (1978): The life and mind of J. Dewey, Southern Illinois University Press, Carbondale.

FELDMANN, W. T. (1934): The Philosophy of J. Dewey, Baltimore.

HOOKS, S. (Ed.) (1950): J. Dewey, philosopher of science and Freedom, New York.

MENDOZA DE MONTORO, A. (1940): Líneas fundamentales de la filosofía de J. Dewey, Buenos Aires.

RATNER, J. (1939): John Dewey's Philosophy, Modern Library, New York.

SCHILPP, P. A. (1951): The Philosophy of J. Dewey, Tudor, New York. Durante mucho tiempo ha sido la obra más clásica sobre la filosofía de John Dewey.

VIRETTO, M. T. (1938): Il pensiero de J. Dewey, Nápoles.

WHITE, M. G. (1943): The origin of Dewey's Instrumentalism, Columbia University Press, New York.

\section{PENSAMIENTO PEDAGÓGICO Y OBRAS FILOSÓFICO-EDUCACIONALES}

\subsection{Pensamiento pedagógico de John Dewey}

La premisa mayor de la que deriva Dewey su ulterior pensamiento filosófico sobre el proceso educativo es el concepto de educación. Estas son sus célebres palabras: «Alcanzaríamos así una definición técnica de la educación: es aquella reconstrucción o reorganización de la experiencia que da sentido a la experiencia y que aumenta la capacidad para dirigir el curso de la experiencia subsiguiente ${ }^{4}$.

En esta definición cabe resaltar: la experiencia, la reconstrucción, la reorganización y la capacitación para seguir experimentando. Llegó a ella como consecuencia de tres postulados filosóficos: la experiencia, la continuidad y la reconstrucción. La

4. J. DEWEY, o. c., pp. 87-88. 
educación es contemplada desde perspectivas diferentes, siendo las más sobresalientes la neurobiológica, la psicológica, la moral y la social ${ }^{5}$. Todas están insinuadas en su obra My pedagogic Creed, publicado en 1897, verdadero manifiesto filosófico educacional del pedagogo norteamericano. Añádanse el primer principio educativo, que no es otro que el interés, el estudio de la escuela como institución social, en consonancia con la vida real, y el progreso social como efecto de la educación formal e institucionalizada.

Dewey fue posiblemente el primer filósofo de la educación que medió entre la concepción escolástica y perennialista y la naturalista, en materia de fines e intencionalidades. Los fines no son extrínsecos al acto educador, ni puede éste ser entendido como espontáneo. Los fines son intrínsecos a la actividad pedagógica; y por esta razón le he calificado de inmanentista. Aunque los perennialistas norteamericanos se disgustaron por esta doctrina, hoy es frecuentemente repetida, desconociendo muchas veces su más genuino representante ${ }^{6}$.

Su axiología le obliga a apreciar el estudio por sí mismo, como un valor intrínseco y, por consiguiente, no instrumental ni subordinado a nada. La ciencia y el currículum escolar son, de esta manera, apreciables y valiosos, incapaces de ser jerarquizados por su mayor o menor entidad?

La moralización, dentro de su doctrina genérica sobre la formación de los hábitos y de la disciplina, es primordial en el proceso educativo. La educación, en esta vertiente, consiste en la adquisición de disposiciones, en la formación de actitudes reflexivas y en la consecución de los conocimientos empíricos, necesarios para decidir lo que ha de hacerse o evitarse. La fusión entre la experiencia y el conocimiento permiten afirmar que el niño se moraliza mediante la instrucción, vieja cuestión debatida resueltamente desde Sócrates a la moderna corriente de Psicología cognitiva.

La Filosofía de la Educación nació con los sofistas griegos y fue mérito de Platón el que los pensadores contemplativos aplicaran sus energías al campo educacional. La Filosofía de la educación es para Dewey una «formulación explícita de los problemas inherentes a la formación de los hábitos mentales y morales adecuados a las dificultades de la vida contemporánea». La filosofía nació y se ha desarrollado en constante aplicación a la formación del hombre. No hay otra filosofía que la Filosofía de la Educación, pues «si una teoría no introduce diferencia en el trabajo educativo, tiene que ser artificial».

\subsection{Principales obras pedagógicas de John Dewey}

DEWEY, J. (1987): My pedagogic creed, University of Chicago Press. Traduc. Castellana: «Mi credo pedagógico», Losada, Buenos Aires, 1944.

- (1899): The school and society, University of Chicago Press. Traduc. Castellana: «La escuela y la sociedad», Beltrán, Madrid, 1929.

- (1899): Lectures in the Philosophy of Education, reeditada recientemente por la Randon House, de New York (1966).

- (1909): Moral principles in education, Greenwood Press, New York.

5. P. Fermoso (1976), Teoría de la Educación, Agulló, Madrid, pp. 25-27.

6. P. Fermoso (1976), o. c., pp. 214-216.

7. J. DEWEY, o. c., p. 261. 
- (1915): Schools of tomorow. Traduc. Castellana: «La escuela de mañana», Losada, Buenos Aires, 1950.

- (1916): Democracy and Education: An introduction to the Philosophy of Education, McMillan, New York. Traduc. Castellana: «Democracia y Educación», Losada, Buenos Aires, 1971. Es su obra maestra sobre Filosofía de la Educación y una de las mejores escritas en la época contemporánea sobre esta disciplina. Puede ser considerada como la definitiva consagración de su autonomía y el inicio de una resonancia y aceptación creciente en el Currículum de las Facultades de Educación.

- (1929): The sources of a science of education, Tiveright, New York. Traduc. Castellana: «Las fuentes de una ciencia de la educación», reproducida en diversas ediciones antológicas de J. Dewey.

- (1938): Experience and Education, McMillan, New York. Traduc. Castellana: «Experiencia y educación», Losada, Buenos Aires, 1960. Una de las aportaciones más ricas de cuantas hizo J. Dewey. Fundamental para interpretar su pensamiento a la luz de su Epistemología.

- (1940): Education Today, Putman, New York. Traduc. Castellana: «La educación hoy», Losada, Buenos Aires, 1965.

- (1948): Philosophy of Education, Littlefield, Totowa (N. J.). Traduc. Castellana: «Filosofía de la Educación», Losada, Buenos Aires, 1954.

- (1974): J. Dewey on Education: Selected writings, University of Chicago Press. Edición e introducción de R. D. Archambault.

- (1985): Democracia i Escola, Eumo, Vic. Es una antología o crestomatía del pensamiento pedagógico de J. Dewey; es plausible que se haya hecho esta edición, al modo como existen otras en Italia, para que los estudiantes puedan disponer de los originales de J. Dewey.

- (1926-1928): Obras de J. Dewey, La Lectura, Madrid. Es una edición antigua hecha en España, para divulgar lo mejor del pensamiento pedagógico de J. Dewey. Comprende siete volúmenes. Algunos reproducen una obra completa; otros, más frecuentemente, están integrados por capítulos de varias obras. La más reproducida fue «Democracia y Educación», que se había publicado en Estados Unidos 10 años antes. Posteriormente fue Lorenzo Luzuriaga quien desde la Editorial Losada, en el exilio, tradujo y publicó las principales obras pedagógicas de J. Dewey. Son ediciones agotadas, de difícil localización en bibliotecas.

He aquí la referencia de los siete volúmenes:

Vol. I: «La escuela y el niño».

Vol. II: «Ensayos de educación»; «Los principios morales que cimentan la educación»; «El interés en relación con el entrenamiento de la voluntad»; «Mi Credo Pedagógico»; «La Psicología y la práctica social».

Vol. III: «Teorías sobre la educación». Es la primera parte de «Democracia y Educación».

Vol. IV: «Los fines, las materias y los métodos de la educación». Es la reproducción de otra parte de «Democracia y educación».

Vol. V: «Filosofía de la educación: los valores educativos». Igualmente capítulos de «Democracia y educación».

Vol. VI: «Cómo pensamos».

Vol. VII: «El hábito y el impulso en la conducta». Es una edición fragmentada de «Naturaleza humana y conducta». 


\section{BIBLIOGRAFÍA SOBRE SU PENSAMIENTO PEDAGÓGICO}

No hemos recogido todas las obras, tesis doctorales y artículos, que sobre la Filosofía de la Educación de John Dewey se han escrito. Ni hemos reproducido páginas del repertorio de J. A. Boydston-K. Poules, inaccesible en España y sin justificación suficiente su adquisición informática o reprográfica. He prescindido de artículos escritos en lenguas no occidentales, porque ni las conozco ni las conocen nuestros lectores, de ordinario. Me he limitado a los idiomas más importantes de nuestra área geográfica y cultural. Las tesis y las tesinas inéditas, que aparecen en algunas notas bibliográficas o florilegios no son tampoco de fácil consulta, porque no siempre se indican los fondos documentales que disponen de ellas. He omitido también, en principio, artículos excesivamente breves, que poco añaden sobre cuanto ampliamente se ha dicho acerca de J. Dewey. A pesar de estas limitaciones, de las que soy consciente, la bibliografía presentada ayudará a docentes e investigadores en Filosofía de la Educación.

\subsection{Repertorios y estudios generales}

a) Repertorios:

ADREANI, S. (1979): Studi italiani su J. Dewey, un contributo bibliográfico», Richerche Pedagogiche, nro. 52-53, pp. 49-60. Recoge la bibliografía italiana desde 1905-1952; es decir, la pertinente a los años anteriores a la muerte de J. Dewey.

BOYDSTON, J. A.; POULES, K. (1974): Checklist of writings about J. Dewey: 1887-1973, Southern Illinois University Press.

THOMAS, M. H. (1939): Bibliography of J. Dewey (1882-1939), University of Columbia, New York.

- (1962): John Dewey: A centenial Bibliography, University of Chicago. Este especialista en la Bibliografía de J. Dewey preparó esta publicación, con motivo del primer centenario del nacimiento del prestigioso filósofo norteamericano (1859-1959).

b) Estudios Generales

ARChambault, R. D. (1968): John Dewey reconsidered, Random House, New York.

ARMANDO, A. (1984): Filosofía e Psicologia nel primo Dewey, La Nuova Italia, Firenze.

BERNSTEIN, R. J. (1966): John Dewey, Washington Square Press, New York.

BHATTACHARYA, N. C. (1968): «J. Dewey's Instrumentalism, Democratic Ideal and Education», Educational Theory, Vol. 18, pp. 60-72.

- (1974): «Demythologizing J. Dewey», Journal of Educational thought, Vol. 8, nro. 3, pp. 117-125.

BOWEN, J.; HOBSON, P. R. (1974): Theories of Education, Wiley and Sons Australasia, Sidney. Traduc. Castellana: «Teorías de la educación», Limusa, México, 1979, pp. 163-214.

CAMERUZZI, M. (1983): La rivoluzione pedagogica di John Dewey, E.S.A., Roma.

CORAllo, G. (1950): La Pedagogia di John Dewey, S.E.I., Torino.

- (1969): Dewey, La Scuola Editrece, Brescia.

CoughlaIN, N. (1975): Young John Dewey, New York.

CHILDS, J. L.; KILPATRICK, W. H. (1965): «John Dewey as educator», The Philosophy of John Dewey, New York. 
Deledalle, G. (1965): La Pédagogie de John Dewey. Philosophie de la continuité, Editions du Scarabée, París.

- (1977): «Pour situer la Pédagogie de Dewey», Educational Development, nro. 115, pp. 4-10.

Du CHEMIN, R. C. (1953): Aspects of the Philosophy of J. Dewey and B. Russell and their relations to Education, Ohio State University.

DwORKIN, M. S. (1959): Dewey on Education, Teachers College of Columbia University, New York. Clásica obra norteamericana, que al igual que otras de títulos similares es una antología de los escritos pedagógicos de J. Dewey, precedidos de una introducción.

ECHEVARRÍA, L. (1952): «John Dewey», Bordón, Vol. 4, pp. 271-273. Los incluimos, a pesar de su brevedad, por ser uno de los pocos títulos escritos en España sobre Dewey.

FEINBERG, W. (1975): «On reading Dewey», History of Education Quarterly, Vol. 15, nro. 4, pp. 305-415.

FranKENA, W. K. (1965): Three historical Philosophies of Education: Aristotle, Kant, Dewey, Scott, Foresman and Co., Illinois. Traduc. Castellana: «Tres filosofías de la educación en la historia: Aristóteles, Kant, Dewey», Uteha, México, 1968, pp. 242-353. Interesante introducción al pensamiento pedagógico de J. Dewey, hecha por un especialista de reconocido prestigio.

GAllaGHER, D. A. (Ed.) (1968): Some philosophers on Education, Marquette University Press, pp. 1-25.

GEIGERS, G. R. (1958): John Dewey in perspective, Oxford University Press, New York.

GRANESE, A. (1966): Il John Dewey, La Nuova Italia Editrice, Firenze.

HENDLEY, B. (1986): Dewey, Russell, Withehead: philosophers as educators, Southern Illinois University, Carbondale.

HESSEN, S. (1953): La Pedagogia di John Dewey, Armando, Roma. Una buena monografía, debida a la pluma de este renombrado teórico italiano.

«John Dewey revisited» (1975), History of Education Quarterly, Vol. 15, nro. 7, pp. 3-85.

LIBRIZZI, M. (1973): Problemática filosofica, psicologia e pedagogica su Dewey, Castorina, Catania.

McKEEVER, C. F. (1956): La Filosofía de la Educación en John Dewey, Universidad Complutense de Madrid. Tesis Doctoral de un norteamericano, en la que sistematizó la Filosofía de la Educación de J. Dewey.

MANTOVANI, J. (1962): Filósofos y educadores, El Ateneo, Buenos Aires. Uno de estos filósofos es J. Dewey.

MAYHEW, K.; EDWARDS, A. C. (1936): The Dewey School. The laboratory school of the University of Chicago: 1896-1903, Appleton-Century, New York.

MORGENBESSER, S. (Ed.) (1977): Dewey and his critics, Hackett, Indianopolis.

NASSIF, R. (1968): John Dewey: su pensamiento pedagógico, C.E.A.L., Buenos Aires. Una monografía escrita por un especialista, de renombre internacional, oriundo de Argentina.

PETERS, R. S. (Ed.) (1977): John Dewey reconsidered, Routledge and Kegan Paul, London. El Editor, R. S. Peters es, posiblemente, el filósofo de la educación inglesa de mayor fama y prestigio. Además de ser el líder de la Escuela de la Educación, de Londres, ha sido el Presidente-Fundador de la Sociedad Inglesa de Filosofía de la Educación y alma de la revista por ella publicada, Journal of Philosophy of Education.

PRICE, K. (1962): Education and philosophical thought, Allyn and Bacon. Entre otros filósofos estudia a J. Dewey.

PUZZANGHERA, E. (1983): La concezione filosofica e pedagogica di J. Dewey, Magalini, Brescia. 
Questioni di Storia della Pedagogia, La Scuola Editrice, Brescia, 1967. Contiene un interesante capítulo sobre J. Dewey.

ROMANI, S. (1984): «Le fonti di una scienza dell'educazione. Reflessioni sulla teoria formale di John Dewey», Pedagogia e Vita, nro. 6, pp. 609-617.

SKILBECK, M. (Ed.) (1970): Dewey, Collier-McMillan, London.

SPADAFORA, G. (1984): Rileggendo la Pedagogia di Dewey, Periferia, Cosenza.

VIRCILLO, D. (1972): Struttura e Educazione del pensiero in Dewey e Bruner, Peloritana, Messina. Esta monografía investiga las aportaciones pedagógicas de Dewey y del psicólogo cognitivo Bruner.

WEBER, Ch. O. (1960): Basic philosophies of Education, Holt, Rinehart and Winston, New York. Esta obra es una de las síntesis más logradas sobre los sistemas filosóficos más relevantes en educación. Exposición acertada sobre J. Dewey.

WITH, A. G. (1966): John Dewey as educator, J. Wiley and Sons, New York.

WYNNE, J. P. (1963): Theories of Education: An introduction to the foundations of Education, Harper and Row, New York.

\subsection{Fundamentación de la teoría educacional}

En la evolución histórica de la Filosofía de la Educación se advierte que antes de 1950 fue frecuente dedicar esfuerzos a las «Foundations», verdaderos manuales universitarios para Centros universitarios de Ciencias de la Educación, en Estados Unidos. Los títulos siguientes contemplan este aspecto en J. Dewey.

AREGERI, G. (1974): Concetti fondamentali sul pragmatisme, sul James e sul Dewey, Fiamma Serafica, Palermo. De los tres sistemas influyentes en Dewey se estudia el Pragmatismo.

BAKER, M. N. C. (1955): Foundations of John Dewey's educational theory, King's Crown Press, New York.

BRUIMBAUGH, R. S.; LAWRENCE, N. M. (1963): Philosophers on Education: Six essays on the foundations of western thought, Houhgton. Uno de los seis ensayos está dedicado a $J$. Dewey.

GILligan, J. (1951): A critical study of J. Dewey Pedagogy and his philosophical fonts, Angelicum, Roma. Interesante tesis doctoral sobre las fuentes filosóficas del pensamiento pedagógico de J. Dewey.

GÖTZ, B. (1973): «Zur wissenschafstheoretischen Bregündung der pragmatischen Erziehungsphilosophie», Pädagogische Rundschau, Vol. 27, nor. 2, pp. 63-90.

WHITE, M. G. (1950): The origin of J. Dewey Instrumentalism, New York. Buena introducción al Instrumentalismo de J. Dewey.

WILSON, P. J. (1975): «Dewey's Hegelianism», History of Education Quarterly, Vol. 15, nro. 1, pp. 87-92. Se estudia el hegelianismo influyente en Dewey a través de Harris y Morris, de la John Hoppkins University (Baltimore).

\subsection{Disciplinas filosóficas y educación}

He indicado ya que casi todas las disciplinas filosóficas fundamentan la concepción deweyana de la educación. Algunos estudios monográficos ayudarán a comprender esta escueta afirmación. 
a) Epistemología y educación

FOX, J. T. (1969): «Epistemology, Psychology and their relevance for education in Bruner and Dewey», Educational Theory, Vol. 19, nro. 1, pp. 58-75. De nuevo el parangón entre Dewey y Bruner. De nuevo la Psicología cognitiva de ambos autores condicionando la educación.

MARín CABRERo, R. (1949): «Los objetos del conocimiento en la Pedagogía de Dewey», Actas del Congreso Internacional de Pedagogía, Santander, Vol. I. pp. 257-264.

b) Lógica y educación

LU, H. C. (1968): «Dewey's logical Theory and his conception of Education», Educational Theory, Vol. 18, nro. 4, pp. 388-395.

c) Ideología y educación

EASTMAN, G. (1967): «The ideologizing of Theories: J. Dewey's educational Theory», Educational Theory, Vol. 17, pp. 103-119.

d) Antropología y educación

FENDRICH, R. (1975): «The problem of anthropocentrism in Dewey's Metaphysics», International Philosophy Quarterly, Vol. 15, nro. 1, pp. 149-159.

HAGENMAIER, Th.; HAGENMAIER, Th. (1968): Neu Aspekte der Reformpädagik: Die anthropologische J. Deweys und ihre Bedeurung für die Pädagogik, Quelle und Meyer, Heidelberg.

e) Etica y educación

CARPITA, A. (1974): «Il problema morale secomdo Dewey», Problemi della Pedagogia, Vol. 20, nros. 4-5, pp. 590-596.

COWARD, B. F. (1967): «J. Dewey's concept of good, Educational Theory, Vol. 17, pp. 8390. Cuestión importante en los filósofos de la educación; también en J. Dewey.

GALlito, A. (1981): Etica e Pedagogia nel pensiero di J. Dewey, EDAS, Messina. Es una de las mejores monografías sobre las relaciones entre la Etica y la educación en J. Dewey.

HARRIGTON, K. W. (1973): «J. Dewey's Ethics and the classical conception of man», Dioti$m a$, Vol. 1, p. 125-148.

MATAIX, A. (1964): La normal moral en J. Dewey, Revista de Occidente, Madrid. Entre las escasas investigaciones españolas sobre Dewey sobresalen las hechas por Mataix acerca de su Etica. Valioso libro para profundizar en el pensamiento pedagógico del ilustre estadounidense.

- (1968): «La norma de la moralidad en J. Dewey», Revista de Filosofía, Vol. 27, pp. 149170.

MIN, P. G. (1972): «Dewey's pragmatic Ethics and manipulation: An response to W. Feinberg and C. Karier», Educational Theory, Vol. 22, pp. 311-324.

PIETIG, J. (1977): «John Dewey and character education», Journal of Moral Education, Vol. 6, nros. 3-4, pp. 170-180.

SMITH, Ph. L. (1986): «Still hoping to construct a good society: Dewey and Ethics», Harvard Educational Review, Vol. 56, nro. 2, pp. 183-186. 


\section{f) Dialéctica y educación}

SHARGEL, E. (1982): «Dewey's Dialecti», Philosophy of Education, pp. 162-174. Esta publicación corresponde a las Actas de la reunión anual de la Sociedad Norteamericana de Filosofía de la Educación.

\subsection{Su influencia en el mundo}

Aunque la proyección del pensamiento pedagógico de J. Dewey ha alcanzado a todas las latitudes, mención especial merecen algunos países, más seducidos que otros por esta gigantesca figura o más al alcance de sus viajes en vida, como conferenciante o profesor invitado. Además de Estados Unidos han sido Japón e Italia las naciones en las que mayor preocupación ha despertado.

ADREANI, A. (1979): «Studi italiani su J. Dewey; un contributo bibliografico», Richerche Pedagogische, nros. 52-53, pp. 49-60. Recoge Bibliografía desde 1905-1952.

BELlatalla, I. (1979): «Lamberto Borghi interprete di J. Dewey: spunti sulla diffusione del pensiero di Dewey in Italia», Ricerca Pedagogica, nro. 50, pp. 37-42.

BLUMENFELD, S. M. (1972): «J. Dewey and Jewish education», Religious Education, Vol. 67, nro. 3, pp. 163-169.

BoRGHI, L. J. (1951): John Dewey e il pensiero pedagogico contemporaneo negli Stati Uni$t i$, La Nuova Italia Editrice, Firenze.

-(1961): L'ideale educativo di J. Dewey, La Nuova Italia Editrice, Firenze.

KOBOYASKI, V. N. (1964): John Dewey in japanese educational thought, University of Michigan, Ann Arbor (Mich.).

KRONISCH, R. (1982): «John Dewey's influence on Jewish educators: the case of Alexander M. Dushkin», Teachers College Record, Vol. 83, nro. 3, pp. 419-434.

MAÑACH, J. (1959): Dewey y el pensamiento americano, Taurus, Madrid.

PASOV, A. H. (1982): «John Dewey's influence on education around the world», Teachers College Record, Vol. 83, nro. 3, pp. 401-418.

\subsection{Estudios monográficos}

Varios son los temas sectoriales sobre los que J. Dewey incidió en sus obras pedagógicas. Son temas habituales en los programas de Filosofía y/o Teoría de la Educación. Muchos de ellos están relacionados expresamente con su filosofía o con su preocupación política, que no fue escasa; otros son más puros e independientes.

a) Democracia y educación

BHATTACHARYA, N. C. (1968): «J. Dewey's Instrumentalism, democratic ideal and education», Educational Theory, Vol. 18, pp. 60-72. Es interesante ver las relaciones entre su Instrumentalismo y sus ideales democráticos.

BoHnSACK, F. (1976): Erziehung zur Demokratie. J. Dewey's Pädagogik und ihre Bedeutung für die Reform unserer Schule, Maier, Ravensburg. Se ha servido del pensamiento de J. Dewey para ofrecer un modelo de escuela y educación democráticas a la República Federal de Alemania.

HORNE, H. H. (1978): The democratic philosophy of Education. Companion to Dewey's Democracy and Education, Greenwood, Westport (Conn.). Horne, militante en la filosofía 
idealista norteamericana hizo un esfuerzo, desde su posición, por entender el pensamiento democrático educacional de Dewey.

b) Experiencia y educación

Es éste un tema troncal en el pensamiento pedagógico de J. Dewey. Es lógico que haya llamado la atención de los investigadores y estudiosos. Además de la obra original de Dewey, pueden ayudar algunas referencias bibliográficas.

BERNSTEIN, R. J. (Ed.) (1960): Dewey on experience. Nature and freedom, Liberal Arts Press, New York.

JIMÉNEZ, F. G. (1958): «Algunos aspectos educativos del concepto de experiencia en Dewey», Revista Española de Pedagogía, Vol. 16, pp. 75-83.

PATANAÉ, L. R. (1974): «Il concetto d'esperienza in H. Dewey», Problemi della Pedagogia, Vol. 20, nros. 4-5, pp. 506-522.

c) Axiología educacional

BHATTACHARYA, N. G. (1975): «Inquiry, Values and Groth: A reassessment of Dewey's Theory of a valuation», Educational Theory, Vol. 25, nro. 1, pp. 92-101.

ERNST, K. W. (1974): «A comparation of Dewey's theory of valuation and Abraham Maslow's theory of value», Educational Theory, Vol. 24, nro. 2, pp. 130-141. Sugestiva comparación entre la axiología de Dewey y de uno de los principales psicólogos humanistas, famoso por su doctrina sobre la formación de la personalidad y de las motivaciones.

FERMOSO, P. (1977): «Axiología y valores en John Dewey», Educadores, nro. 92, pp. 231248. En este artículo amplié mi interpretación de la axiología de J. Dewey, que ya había expuesto, en síntesis en el Congreso Nacional de Pedagogía (Madrid, 1976).

GoUILOCK, J. (1972): John Dewey's Philosophy of value, New York.

PIтомво, M. I. (1974): Conhecimiento, valor e educaçao em J. Dewey, Pieneiro Editora, Sao Paolo (Brasil). Efectivamente, la Epistemología en Dewey es inseparable de la Axiología; y ambas condicionan la educación. Interesante monografía.

WOLSTEIN, B. (1949): Experience and valuation: A study in J. Dewey's naturalism, New York.

d) La educación social

En su obra «Democracia y Educación» no oculta Dewey sus intenciones, al proponerse idear una alternativa al sistema escolar y educacional norteamericano vigente, a fin de garantizar las libertades y las ansias de igualdad en los Estados Unidos y en su Constitución. Ni la Etica, ni la Pedagogía pueden ser entendidas al margen de lo social. Es su peculiar punto de vista, que no coincide con el sociologismo francés de E. Durkheim.

APPEL, H. J. (1979): Teoría de la escuela en una sociedad industrial democrática, Atenas, Madrid. Dedicada al pensamiento de J. Dewey.

BOURKE, P. F. (1975): «Philosophy and social criticism: John Dewey (1910-1920)», History of Education Quarterly, Vol. 15, nro. 1, pp. 3-16.

BROSIO, R. A. (1967): «Dewey's concept of community: a last third of the twentieth century perspective», Journal of Educational Thought, Vol. 10, pp. 83-90.

DOLL, N. E. (1972): An analysis of John Dewey's educatinal writings interpreted with the reference to this concept of change, John Hopkins University, Baltimore. 
D'URSO, S. (1978): «A evaluation of Dewey's social intelligence», Educational Theory, Vol. 28, pp. 120-130.

EISELE, J. C. (1975): «J. Dewey and the inmigrante», History of Education Quarterly, Vol. 15, nro. 1, pp. 67-85.

FEINBERG, W. (1969): «The conflict between intelligence and Community in Dewey's educational philosophy», Educational Theory, Vol. 19, pp. 236-248.

GALLANT, Th. F. (1972): «Dewey social Reconstructionism and institutional neutrality», Educational Theory, Vol. 22, pp. 427-433.

GOLDSTEIN, J. (1973): «J. Dewey's city on a Hill: The school as model of community», Journal of Educational Thought, Vol. 7, nro. 2, pp. 73-80.

GREEN, J. L. (1979): «Dewey, Russell and the integration of the social», Educational Theory, Vol. 29, nro. 4, pp. 285-296.

HowlETT, Ch. F. (1982): «The prasmatist as pacifist: John Dewey's views on peace education», Teachers College Record, Vol. 83, nro. 3, pp. 435-452.

SCHULTZ, F. M. (1971): «Community as pedagogical enterprise and the functions of schooling with it in philosophy of J. Dewey», Educational Theory, Vol. 21, pp. 320-337.

WIRTH, A. G. (1982): «J. Dewey on relations between industrial democracy and education», Philosophy of Education, pp. 273-285.

\subsection{Relaciones del pensamiento de Dewey y otros sistemas filosóficos}

Dos son las posibles relaciones entre el pensamiento pedagógico de J. Dewey y otras concepciones filosóficas o educacionales. Por una parte, J. Dewey fue fruto de la convergencia de varios sistemas filosóficos y científicos -idealismo, pragmatismo, evolucionismo darwiniano-, y, por otra, su fuerte personalidad y la alternativa por él ofrecida se han impuesto, por su prestigio y fuerza interna, a muchos estudiosos de la Filosofía/Teoría de la Educación.

GREENE, M. (1978): «Dewey, Phenomenology and the matther of consciousness», Philosophy of Education, pp. 454-461. De nuevo Dewey es objeto de una contribución presentada en la reunión anual de la Sociedad Norteamericana de Filosofía de la Educación.

GUMBERT, E. B. (1972): «J. Dewey and the new Liberalism: reactions to the URSS», Educational Theory, Vol. 22, nro. 3, pp. 344-359.

KARIER, C. J. (1975): «John Dewey and the new Liberalism: some reflections and responses», History of Education Quarterly, Vol. 15, nro. 4, pp. 417-443. El liberalismo inglés tuvo destacado papel en la cosmovisión típicamente norteamericana, en el s. XIX. El Neoliberalismo, suavizado ante el colectivismo socialista, encontró un claro exponente en J. Dewey.

KostembauM, V. (1979): «Phenomenology and Dewey's Empirism: A response to L. Troutner», Educational Theory, Vol. 29, pp. 311-324.

SCHEFFLER, I. (1956): «Educational Liberalism and Dewey's Philosophy», Harvard Educational Review, Vol. 26, nro. 2, pp. 190-196.

TROUTNER, L. F. (1972): «The Dewey-Heidegger comparison revisisited: A perspectival parternship for Education», Philosophy of Education, pp. 28-44.

WIRTH, A. G. (1974): «Philosophical issues in the cocational liberal studies controversy (1900-1917): J. Dewey versus the social efficiency philosophers», Studies in Philosophy of Education, Vol. 8, nro. 3, pp. 169-182. 


\subsection{Varios}

En este último apartado reuno un puñado de investigaciones monográficas, que no han tenido cabida en las cuestiones más destacadas en la exposición del pensamiento pedagógico de J. Dewey, dada su variedad y dispersión.

BOURDIEAUX, R. M. (1972): «J. Dewey's concept of functional self», Educational Theory, Vol. 22, nro. 3, pp. 334-343.

BURNETT, J. R. (1979): «Whatever happened to J. Dewey», Teachers College Record, Vol. 81, pp. 192-210.

CASTELlazo, M. S. (1984): «John Dewey e il lavoro nella scuola: attualitá del suo pensiero», I Problemi della Pedagogia, Vol. 30, nro. 3, pp. 251-262.

DENNIS, L. J. and others (1981): «Mead and Dewey. Thematic connections on educational topics», Educational Theory, Vol. 31, pp. 319-331.

- (1972): «Dewey's debt to Albert Coombs Barnes», Educational Theory, Vol. 22, nro. 3, pp. 325-333.

KLAFKI, W. (1978): «Die Aktualität der Pädagogik J. Deweys», Zeitschrift für Pädagogik, Vol. 24, nro. 5, pp. 781-793.

LAWSON, A. (1975): «John Dewey and the hope to reform», History of Education Quarterly, Vol. 15, nro. 1, pp. 31-66.

MAZETTI, R. (1970): Dewey a Bruner: il processo educativo, Armando, Roma. Otro nuevo empeño en establecer comparaciones entre J. S. Bruner y J. Dewey. En el presente caso, sobre un tema general, el proceso educativo.

Molinos, Ma. del C. (1983): Concepto y práctica del Currículum en J. Dewey, Universidad de Navarra, tesis doctoral inédita.

POOLE, R. H. (1975): «The real failure of John Dewey», Educational Review (Gran Bretaña), Vol. 27, nro. 2, pp. 138-149. Desde Inglaterra se habla del fracaso de J. Dewey. Resulta alarmante el título de este artículo, tras comprobar la aceptación de J. Dewey en diversas partes del mundo.

WAIN, K. (1984): «Lifelong Education: A deweyian challenge», Journal of Philosophy of Education, Vol. 18, nro. 2, pp. 257-264.

WELDON, W. (1973): «Dewey's conception of the environment as an educative force», Journal of Environmental Education, Vol. 4, nro. 4, pp. 56-58.

WILBANKS, J. J. (1974): «Educational reductionism», Educational Theory, Vol. 24, nro. 1, pp. 73-94.

WIRTTH, A. G. (1972): «J. Dewey's philosophical opposition to Smith-Hughes type vocational education», Educational Theory, Vol. 22, nro. 1, pp. 73-84.

- (1978): «The deweyian tradition as alternative to systems efficiency and romantic freedom», Journal of Thought, Vol. 13, nro. 4, pp. 273-278.

ZERBY, C. L. (1975): «John Dewey and the polish question», History of Education Quarterly, Vol. 15, nro. 1, pp. 3-16. 